\title{
POVERTY, PRIVILEGE, AND BRAIN DEVELOPMENT: \\ EMPIRICAL FINDINGS AND ETHICAL IMPLICATIONS
}

\author{
Martha J. Farah, Kimberly G. Noble and Hallam Hurt \\ University of Pennsylvania
}

\begin{abstract}
Introduction
After my daughter was born people sometimes asked me "Has having a child gotten you interested in brain development?" My answer was "No, not exactly, but having babysitters has." More specifically, getting to know my daughter's babysitters and becoming close with some of them and their families called my attention to a set of complex social problems that are, I believe, partly related to brain development. Up until this time I had viewed cognitive neuroscience as an ivory tower pursuit, whose potential applications were few and far between (except, of course, in NIH grant proposals). I now believe that cognitive neuroscience can provide a framework for understanding and even solving certain societal problems. One such problem is the persistence of poverty across generations. This chapter summarizes recent research I have undertaken with my co-authors on the relation between poverty and children's
\end{abstract}


cognitive achievement, which is in turn related to their future socioeconomic prospects.

My babysitters were mostly of low socioeconomic status (SES). These women had little education, little experience in jobs other than domestic work, and relied on state assistance supplemented by cash babysitting wages to support themselves and their children. Although they had one foot in the middle class world through their babysitting jobs, the differences between their lives and my middle class existence were nevertheless immense. Nowhere were the differences more dramatic than in the realm of child development. Their daughters and sons and nieces and nephews began life with the same evident promise as my daughter and her friends. Yet as the years went on, I saw their paths diverge.

On our side of the tracks, preschoolers know their letters and numbers, elementary school children are familiar with different neighborhoods and different cities, and middle school children read newspaper headlines and ask their parents about them. Not so with the children I met on their side of the tracks. However, the low SES children did show a kind of sad precocity compared with middle class children in their first-hand experience with violence, death and the criminal justice system. Preschoolers growing up in the inner city know to fear the neighbors from the local crack house. By elementary school they recognize the sound of gun shots and by middle school they know about prison because a 
relative has been there. It seemed to me that children's experience of the world is very different in low and middle SES environments. Most middle SES children have abundant opportunities to explore the world, literally, in terms of people met and places seen, and figuratively, in terms of the world of ideas. In contrast, low SES children generally have fewer interactions with the wider world and much of what they do experience is stressful. Basic research with animals has established the powerful effects of both environmental impoverishment and stress on the developing brain.

The research reported in this chapter is an attempt to relate findings on socioeconomic status and brain development. The ultimate goals of this work are to inform practical decisions concerning child policy, and to reveal the neuroethical dimensions of the problem of childhood poverty.

(MF)

Socioeconomic status and child development There is a gulf between low and middle SES children in their performance on just about every test of cognitive development, from the Bayley Infant Behavior Scales to IQ and school achievement tests. Furthermore, these SES disparities are not subtle. For example, in one study we conducted, the average IQ of a group of healthy low SES 6 year-olds was 81. Only $20 \%$ of the sample scored in the normal range, defined as 90 or above (Hurt et al. 
1998). Looking further ahead in the developmental trajectories of poor and middle class children, a $\$ 10,000$ increment in family income was associated with a $16 \%$ increase in high school graduation rates for children of middle income families, but a $600 \%$ (six hundred) increase in high school graduation for low income and poor children (Duncan et al. 1998).

The gap in cognitive achievement between children of low and middle SES suggests one possible answer to the question of why poverty persists. This question has occupied sociologists since their field began, and has been answered in many ways. Marx (1867) emphasized purely economic factors that create and maintain social stratification. Functionalist accounts highlight the many ways in which society as a whole is served by the enduring presence of a lower class (e.g., Weber 1923). The concept of a Culture of Poverty (Lewis 1965) emphasizes causes within individuals and their subculture, rather than external societal forces, in perpetuating poverty across generations. Each account undoubtedly captures some truth about the complex and multifactorial processes that confine children born of poor parents to lifelong poverty. Cognitive neuroscience offers yet another perspective on the problem. Without good intellectual development and educational attainment, people are denied all manner of economic and social opportunities. If cognitive neuroscience can help us understand the SES gap in cognitive achievement, it will have provided an important new perspective on the problem of poverty.

For the sake of exploring the cognitive neuroscience perspective on transgenerational poverty, and discovering what, if anything, it can contribute to correcting socioeconomic inequality, the first order of business is to ask whether 
socioeconomic status bears any straightforward relation to brain development. On the face of things it might seem unlikely that characteristics such as income, education and job status, which are typically used to estimate SES, would bear any systematic relationship to physiological processes such as those involved in brain development. It is, however, well established that SES affects physical health through a number of different causal pathways (Adler et al. 1994), many of which could play a role in brain development. It is also clear that poverty is associated with differences in brain function on the basis of the differences in standardized test performance cited earlier, as cognitive tests reflect the function of the brain. However, for a cognitive neuroscience approach to be helpful, the relations between socioeconomic status and the brain must be relatively straightforward and generalizable. The first question that my collaborators and I addressed was therefore: Can we generalize about the neurocognitive correlates of socioeconomic status? Once we have established the neurocognitive profile of childhood poverty, we can begin to test more specific hypotheses about causal mechanisms.

The neurocognitive profile of childhood poverty: Three studies In an initial study we compared the neurocognitive performance of 30 low and 30 middle SES AfricanAmerican Philadelphia public school kindergarteners (Noble, Norman \& Farah 2005). The children were tested on a battery of tasks adapted from the cognitive neuroscience literature, designed to assess the functioning of five key neurocognitive systems. These systems are described briefly here. 
- The Prefrontal/Executive system enables flexible responding in situations where the appropriate response may not be the most routine or attractive one, or where it requires maintenance or updating of information concerning recent events. It is dependent on prefrontal cortex, a late-maturing brain region that is disproportionately developed in humans.

- The Left perisylvian/Language system is a complex, distributed system encompassing semantic, syntactic and phonological aspects of language and dependent predominantly on the temporal and frontal areas of the left hemisphere that surround the Sylvian fissure.

- The Medial temporal/Memory system is responsible for one-trial learning, the ability to retain a representation of a stimulus after a single exposure to it (which contrasts with the ability to gradually strengthen a representation through conditioning-like mechanisms), and is dependent on the hippocampus and related structures of the medial temporal lobe.

- The Parietal/Spatial cognition system underlies our ability to mentally represent and manipulate the spatial relations among objects, and is primarily dependent upon posterior parietal cortex.

- The Occipitotemporal/Visual cognition system is responsible for pattern recognition and visual mental imagery, translating image format visual representations into more abstract representations of object shape and identity, and reciprocally translating visual memory knowledge into image format representations (mental images). 
Not surprisingly, in view of the literature on SES and standardized cognitive tests, the middle SES children performed better than the low SES children on the battery of tasks as a whole. For some systems, most notably the Left perisylvian/Language system and the Prefrontal/Executive system, the disparity between low and middle SES kindergarteners was both large and statistically significant. Indeed, the groups differed by over a standard deviation in their performance composite on language tests, and by over two thirds of a standard deviation in the executive function composite. The other systems did not differ significantly between low and middle SES children, and in fact differed significantly less than the first two.

In a subsequent study we attempted to replicate and extend these findings in a larger group of children, 150 first graders of varying ethnicities whose SES spanned a range from low through middle (Noble, McCandliss \& Farah 2004; "Socioeconomic gradients predict individual differences in neurocognitive abilities," submitted). These children completed a different set of tests designed to tap the same neurocognitive systems as the previous study, with two main differences: First, instead of considering "prefrontal/executive" to be a single system, we subdivided it into three subsystems each with its own tests.

- The Lateral prefrontal/Working memory system enables us to hold information "on line" to maintain it over an interval and manipulate it, and is primarily dependent on the lateral surface of the prefrontal lobes. (Note that this is distinct from the ability to commit information to long-term memory, which is dependent on the medial temporal cortex.) 
- The Anterior cingulate/Cognitive control system is required when we must resist the most routine or easily available response in favor of a more taskappropriate response, and is dependent on a network of regions within prefrontal cortex including the anterior cingulate gyrus.

- The Ventromedial prefrontal/Reward processing system is responsible for regulating our responses in the face of rewarding stimuli, allowing us to resist the immediate pull of a attractive stimulus in order to maximize more longterm gains.

A second noteworthy difference between this and the previous study concerned the tests of the Medial temporal/Memory system. In both of the tasks used to assess memory in the previous study, the test phase followed immediately after the initial exposure to the stimuli and memory per se may not have been the limiting factor in performance. The tasks that we used in the second study included a longer delay between initial exposure to the stimuli to be remembered and later test.

As before, the language system showed a highly significant relationship to SES, as did executive functions including Lateral prefrontal/Working memory and Anterior cingulate/Cognitive control components and the Parietal/Spatial cognition system. With a more demanding delay between exposure and test in the memory tasks, we also found a difference in the Medial temporal/Memory system. Performance on the Parietal/spatial system tests also differed as a function of SES.

Finally, we assessed these same neurocognitive systems in older children. We tested 60 middle school students, half of low and half of middle SES, matched for age, 
gender and ethnicity (Farah et al. 2004; submitted). Again, sizeable and significant SES disparities were observed for language and the two executive subsystems, working memory and cognitive control, as well as for memory. SES was not associated with significant differences in the Parietal/Spatial cognition system, the Occipitotemporal/Visual cognition system, or the Ventromedial prefrontal/Reward processing system. In sum, although the outcome of each study was different, there were also commonalities among them despite different tasks and different children tested at different ages. The most robust neurocognitive correlates of SES appear to involve the Left perisylvian/Language system, the Medial temporal/Memory system (insofar as SES effects were found in both studies that tested memory with an adequate delay) and the Prefrontal/Executive system, in particular its Lateral prefrontal/Working memory and Anterior cingulate/Cognitive control components. Children growing up in low SES environments perform less well on tests that tax the functioning of these specific systems.

The profile of SES disparities, with greatest disparity in systems needed for language, memory, working memory and cognitive control, would be expected to affect children's life trajectories. The importance of language and memory is obvious, from the social sphere to the world of school and work. Less obvious is the impact of working memory and cognitive control on real world success, but studies have linked individual differences in these systems to individual differences in children's behavioral self-regulation and adult intelligence and problem-solving ability (Davis et al. 2002; Duncan et al. 1995; Engle et al. 1999; Gray et al. 2003) 
Disentangling cause and effect in the neurocognitive correlates of SES

Do these associations reflect the effects of SES on brain development, or the opposite direction of causality? Perhaps families with higher innate language, executive and memory abilities tend to acquire and maintain a higher SES. Such a mechanism seems likely, a priori, as it would be surprising if genetic influences on cognitive ability did not, in the aggregate, contribute to individual and family SES. However, it seems also seems likely that causality operates in the opposite direction as well, with SES influencing cognitive ability through childhood environment. Note that the direction of causality is an empirical issue, not an ethical one. The issue of whether and to what extent SES differences cause neurocognitive differences or visa versa should not be confused with the issue of whether we have an obligation to help children of any background become educated, productive citizens.

Given that the direction of causality is an empirical issue, what data bear on the issue? The methods of behavioral genetics research can, in principle, tell us about the direction of causality in the association between SES and the development of specific neurocognitive functions. However, these methods have yet to be applied to that question. They have been applied to a related question, namely the heritability of IQ and SES. Cross-fostering studies of within- and between -SES adoption suggest that roughly half the IQ disparity in children is experiential (Capron \& Duyme, 1989; Schiff \& Lewontin, 1986). If anything, these studies are likely to err in the direction of underestimating the influence of environment because the effects of prenatal and early postnatal environment are included in the estimates of genetic influences in adoption studies. A recent twin study by Turkheimer and colleagues (2003) showed that, within 
Iow SES families, IQ variation is far less genetic than environmental in origin. Additional evidence comes from studies of when, in a child's life, poverty was experienced. Within a given family that experiences a period of poverty, the effects are greater on siblings who were young during that period (Duncan et al. 1994), an effect that cannot be explained by genetics. In sum, multiple sources of evidence indicate that SES does indeed have an effect on cognitive development, although its role in the specific types of neurocognitive system development investigated here is not yet known.

\section{Potential causes, physical and psychological}

What aspects of the environment might be responsible for the differences in neurocognitive development between low and middle SES children? A large set of possibilities exist, some affecting brain development by their direct effects on the body and some by less direct psychological mechanisms. Three somatic factors have been identified as significant risk factors for low cognitive achievement by the Center for Children and Poverty (1997): inadequate nutrition, substance abuse (particularly prenatal exposure), and lead exposure.

American children living in urban poverty are at risk for iron deficiency and mildto-moderate protein-energy malnutrition (PEM), which involves shortages of both protein and calories. The neurocognitive impact of these deficiencies is not well established (see Ricciuti 1993 and Sigman1995 for opposing viewpoints). The Center on Hunger, Poverty and Nutrition Policy (1998) has suggested that mild-to-moderate PEM probably has little effect on its own. Iron-deficiency anemia afflicts about one quarter of low-income children in the US (CHPNP 1998) and is known to impair brain 
development when severe. The role of nutrition in SES disparities in brain development has been difficult to resolve because nutritional status is so strongly correlated with a host of other family and environmental variables likely to impact neurocognitive development, including all of the potential mechanisms of causation to be reviewed here. Although nutritional supplementation programs could in principle be used as an "experimental manipulation" of nutritional status alone, in practice these programs are often coupled with other, non-nutritional forms of enrichment or affect children's lives in non-nutritional ways which perpetuate the confound (e.g., children given school breakfast are absent and late less often). The consensus regarding the role of nutrition in the cognitive outcomes of poor children has shifted over the past few decades, from primary cause to a factor that contributes indirectly and through synergizies with other environmental disadvantages (CHPNP 1998).

Lead is a neurotoxin released by peeling paint, which accumulates in the bodies of low-SES children who are more likely to live in old and poorly maintained housing stock. A meta-analysis of low-level lead exposure on IQ indicates estimated that every $10 \mathrm{ug} / \mathrm{dL}$ increase in lead is associated with a 2.6 point decrease in IQ (Schwartz 1994). As with nutrition, the effect of lead synergizes with other environmental factors and is more pronounced in low SES children (Bellinger et al. 1987). For example, low iron stores render children more susceptible to environmental lead (CHPNP 1998).

Prenatal substance exposure is a third factor that affects children of all SES levels but is disproportionately experienced by the poor. Maternal use of alcohol, tobacco, marijuana and other drugs of abuse have been associated with adverse cognitive outcomes in children (Chasnoff et al. 1998). Although the highly publicized 
phenomenon of "crack babies" led to predictions of a generation of irreparably braindamaged children growing up in the inner city, in retrospect this was overreaction. Indeed epidemiological studies have found the effects on cognitive performance to be subtle (Hurt et al. 1999; Mayes 2002; Vidaeff and Mastrobattista 2003). For example, the low SES 4-year olds of Hurt's cohort, whose average IQ was 81, served as control subjects for a cohort with prenatal cocaine exposure, whose average IQ was a statistically indistinguishable 79 . This lack of difference contrasts with the substantial difference between both low SES groups' scores and those of typical middle SES children.

The set of potentially causative somatic factors just reviewed is far from complete. There are SES gradients in a wide variety of physical health measures, many of which could affect children's neurocognitive development through a variety of different mechanisms (Adler et al. 1997). Having briefly reviewed the most frequently discussed factors, we turn now to a consideration of the psychological differences between the experiences of low and middle SES children that could also affect neurocognitive development.

As with potential physical causes, the set of potential psychological causes for the SES gap in cognitive achievement is large, and the causes are likely to exert their effects synergistically. Here we will review research on differences in cognitive stimulation and stress.

One difference between low and middle SES families that seems predictable, even in the absence of any other information, is that low SES children are likely to receive less cognitive stimulation than middle SES children. Their economic status 
alone predicts that they will have fewer toys and books and less exposure to zoos, museums and other cultural institutions because of the expense of such items and activities. This is indeed the case (Bradley et al. 2001a) and has been identified as a mediator between SES and measures of cognitive achievement (Bradley and Corwyn 1999; Brooks-Gunn and Duncan 1997; McLoyd 1998). Such a mediating role is consistent with the results of neuroscience research with animals. Starting many decades ago (e.g., Volkmar \& Greenough, 1972) researchers began to observe the powerful effects of environmental stimulation on brain development. Animals reared in barren laboratory cages showed less well developed brains by a number of different anatomical and physiological measures, compared with those reared in more complex environments with opportunities to climb, burrow and socialize (see van Praag et al 2000 for a review).

Other types of cognitive stimulation are also less common in low SES homes, for example parental speech designed to engage the child in conversation (Adams 1998). The average number of hours of 1-on-1 picture book reading experienced by children prior to kindergarten entry has been estimated at 25 for low SES children and between 1000 and 1700 for middle SES children (Adams 1990). Thus, in addition to material limitations, differing parental expectations and concerns also contribute to differences in the amount of cognitive stimulation experienced by low and middle SES children.

The lives of low SES individuals tend to be more stressful for a variety of reasons, some of which are obvious: concern about providing for basic family needs, dangerous neighborhoods, and little control over one's work life. Again, research bears out this intuition: Turner and Avison (2003) confirmed that lower SES is associated with 
more stressful life events by a number of different measures. The same appears to be true for children as well as adults, and is apparent in salivary levels of the stress hormone cortisol (Lupien et al. 2001).

Why is stress an important consideration for neurocognitive development? Psychological stress causes the secretion of cortisol and other stress hormones, which affect the brain in numerous ways (McEwen 2000). The immature brain is particularly sensitive to these effects. In basic research studies of rat brain development, rat pups are subjected to the severe stress of prolonged separation from the mother and stress hormone levels predictably climb. The later anatomy and function of the brain is altered by this early neuroendocrine phenomenon. The brain area most affected is the medial temporal area needed for memory, although prefrontal systems involved in the regulation of the stress response are also impacted (Meaney et al. 1996).

In search of mechanisms: A preliminary study

The three studies summarized earlier show an association between SES and the development of specific neurocognitive systems, namely language, memory and executive function. Whereas we previously knew that SES was associated with cognitive achievement as measured by broad-spectrum tests of cognitive ability such as $\mathrm{IQ}$ and school achievement tests, our results re-describe this relationship in terms of the theoretically more meaningful components of cognitive function specified by cognitive neuroscience. However, we are still left with a description rather than an explanation.

Nevertheless, appropriately describing a phenomenon can be a crucial step in understanding it. Explanations are facilitated when the phenomenon to be explained is 
described in terms corresponding to the natural kinds involved in potential mechanisms. Knowing that SES effects are manifest in IQ and high school graduation rates tells us little about the possible brain mechanisms of SES effects on cognitive achievement. In contrast, knowing that SES effects are found in specific neurocognitive systems enables us to harness what we know about the development of those systems to frame hypotheses about the origins of the effects. An important corollary of this point is that different mechanisms may be responsible for SES effects on different neurocognitive systems. By resolving the SES disparity into its multiple underlying components, we can disentangle multiple causal pathways and test hypotheses about each separately. This is important because such separation allows more selective, and hence more powerful, tests of mechanism.

The latest phase of our research is an attempt to make use of the description of the SES disparities in neurocognitive development in testing hypotheses about the causal pathways. Drawing on our previous research that identified three neurocognitive systems as having the most robust differences as a function of SES (Perisylvian/Language, Medial temporal/Memory, and Prefrontal/Executive), we are now testing hypotheses concerning the determinants of individual differences in the development of these systems in children of low SES. Specifically, we are investigating the role of childhood cognitive stimulation and social/emotional nurturance (Farah et al. 2005; Childhood experience and neurocognitive development: Dissociation of cognitive and emotional influences).

The participants in this research are 50 low SES middle school students from a cohort of children enrolled at birth in a study of the effects of prenatal cocaine exposure 
(see Hurt et al. 1995). Approximately half of the children have been exposed to cocaine prenatally and half have not. Maternal use cocaine as well as amphetamines, opiates, barbiturates, benzodiazepines, marijuana, alcohol and tobacco are ascertained by interview and medical record review at time of birth and, for all but the last three, maternal and infant urine specimens.

As part of the ongoing study of these children, a research assistant visited the home of each child at ages 4 and 8 and administered the HOME (Home Observation and Measurement of Environment, Caldwell \& Bradley, 1984). The HOME includes an interview with the mother about family life and observations of the interactions between mother and child. The HOME has a number of different subscales relevant to different aspects of the child's experience. We combined a number of different subscales indicative of the amount of cognitive stimulation provided to the child to make a composite measure of cognitive stimulation, and a number of different subscales indicative of the amount of social/emotional nurturance provided to the child to make a composite measure of social/emotional nurturance. The subscales used for each composite, along with representative items, were as follows:

- The cognitive stimulation composite for 4 year-olds was composed of: Learning stimulation ("child has toys which teach color," "at least 10 books are visible in the apartment"), language stimulation ("child has toys that help teach the names of animals," “mother uses correct grammar and pronunciation,"), academic stimulation ("child is encouraged to learn colors," "child is encouraged to learn to read a few words"), modeling ("some delay of food 
gratification is expected," "parent introduces visitor to child"), and variety of experience ("child has real or toy musical instrument," "child's art work is displayed some place in house"). For 8 year-olds, the subscales used for the cognitive stimulation composite were: Growth fostering materials and experiences ("child has free access to at least ten appropriate books," "'house has at least two pictures of other type of art work on the walls"), provision for active stimulation ("family has a television, and it is used judiciously, not left on continuously," "family member has taken child, or arranged for child to go to a scientific, historical or art museum within the past year"), family participation in developmentally stimulating experiences ("Family visits or receives visits from relatives or friends at least once every other week," "family member has taken child, or arranged for child to go, on a trip of more than 50 miles from his home").

- The social/emotional nurturance composite for 4 year-olds: was composed of: Warmth and affection ("parent holds child close 10-15 minutes per day," "parent converses with child at least twice during visit") and acceptance ("parent does not scold or derogate child more than once," "parent neither slaps nor spanks child during visit"). For 8 year-olds, the subscales used were: Emotional and verbal responsivity ("Child has been praised at least twice during past week for doing something," "parent responds to child's questions during interview"), encouragement of maturity ("family requires child to carry out certain self care routines," "parents set limits for child and 
generally enforce them"), emotional climate ("parent has not lost temper with child more than once during previous week," "parent uses some term of endearment or some diminutive for child's name when talking about child at least twice during visit") and paternal involvement.

Two other variables with the potential to account for differences in neurocognitive development included in our analyses were maternal intelligence and prenatal substance exposure. The former was measured by the Weschler Adult Intelligence Scale-Revised (WAIS-R). Maternal IQ could influence child neurocognitive outcome by genetic mechanisms or by its effect on the environment and experiences provided by the mother for the child. Prenatal substance exposure was coded for analysis on an integer scale of $0-4$, with one point for each of the following substances: tobacco, alcohol, marijuana and cocaine. Use of other substances was an exclusionary criterion.

We used statistical regression to examine the relations between the neurocognitive outcome measures and the predictor variables cognitive stimulation, social/emotional nurturance, maternal IQ and polysubstance use, as well as the child's gender and age at time of neurocognitive testing. Our results indicate that the development of different neurocognitive systems are affected by different variables.

Children's performance on the tests of Left perisylvian/Language was predicted by average cognitive stimulation. This was the sole factor identified as predicting language ability by forward stepwise regression, and one of three factors identified by backwards stepwise regression, along with the child's gender and the 
mother's IQ. In contrast, performance on tests of Medial temporal/Memory ability was predicted by average social/emotional nurturance. This was the sole factor identified as predicting memory ability by forward stepwise regression and one of three factors identified by backwards stepwise regression, along with the child's age and cognitive stimulation. The relation between memory and early emotional experience is consistent with the animal research cited earlier, showing a deleterious effect of stress hormones on hippocampal development. Our analyses did not reveal any systematic relation of the predictor variables considered here to Lateral prefrontal/Working memory or Anterior cingulate/Cognitive control function. In conclusion, different aspects of early experience affect different systems of the developing brain. Cognitive stimulation influences the development of language, whereas social/emotional nurturance affects the development of memory but not language.

Conclusions: Brain plasticity and human potential

Change, continuity, and personal identity are core problems in bioethics. They arise when we ask at what stage of human development the organism is a person, whether to respect the current or prior wishes of a demented individual, and whether drugs like Ritalin or Prozac undermine the "authenticity" of healthy people using them for enhancement. What each of these examples has in common is that a biological process - development, disease or drug action - results in a corresponding mental change. We are left pondering how to apply the fundamentally static, categorical concepts of person, competency and identity following these physical shifts. 
The research summarized here concerns another manifestation of this core neuroethical conundrum. It reminds us that who we are is determined not only by genetically programmed development, neurodegenerative disease and psychoactive drugs, as in the familiar neuroethical examples just noted, but also by the socioeconomic circumstances of our childhoods in equivalently physical, mechanistic ways. Neuroethicists have rightly called attention to the ethically problematic ability of drugs to change who we are, for example giving us increased self-esteem or self-control by the effects of certain molecules on certain receptors (Fukuyama, 2002; Kass, 2003). It is metaphysically just as perplexing, and socially at least as distressing, that an impoverished and stressful childhood can diminish us by equally concrete physical mechanisms, such as the impact of early life stress on medial temporal memory ability through neuroendocrine mechanisms.

What are the implications for society of a more mechanistic understanding of the effects of childhood poverty on brain development? To different degrees, and in different ways, we regard children as the responsibility of both parents and society. Parents' responsibility begins before birth and encompasses virtually every aspect of the child's life. Society's responsibility is more circumscribed. In the United States, for example, society's contribution to the cognitive development of children begins at age 5 or 6 , depending on whether public kindergarten is offered. The physical health and safety of all infants and children is a social imperative, however, well before school age. Laws requiring lead abatement in homes occupied by children exemplify our societal commitment to protect them from the neurological damage caused by this neurotoxin. Research on the effects of early life stress and limited cognitive stimulation has begun 
to show that these concomitants of poverty have negative effects on neurological development too, by mechanisms no less concrete and real. Thus, neuroscience may recast the disadvantages of childhood poverty as a bioethical issue rather than merely one of economic opportunity.

\section{References}

Adams, B. N. (1998). The Family: a Sociological Interpretation. 1998, Harcourt Brace.

Adams, M. J. (1990). Learning to read: Thinking and learning about print. Cambridge, MA: MIT press

Adler, N. E., Boyce, T., Chesney, M. A., Cohen, S., Folkman, S., Kahn, R. L., \& Syme, S. L. (1994). Socioeconomic status and health: The challenge of the gradient. American Psychologist, 49, 15-24.

Bellinger, D., Leviton, A., Waternaux, C., Needleman, H., \& \& Rabinowitz, C. (1987). Longitudinal analyses of prenatal and postnatal lead exposure and early cognitive development. New England Journal of Medicine,_316, 1037-1043.

Bradley, R. H., \& Corwyn, R. F. (1999). Parenting. In C. L. B. Tamis-Lemonda (Ed.), Child Psychology: A Handbook of Contemporary Issues (pp. 339-362). New York: Psychology Press. 
Bradley, R. H., Corwyn, R.F., McAdoo, H. P. \& Garcia Coll C. (2001a) "The home environments of children in the United States. Part 1: variations by age, ethnicity, and poverty-status. Child Development, 72, 1844-67.

Brooks-Gunn, J., \& Duncan, G. J. (1997). The effects of poverty on children. The Future of Children, 7, 55-71.

Caldwell, B.M., \& Bradley, R.H. (1984). Home Observation for Measurement of the Environment (HOME). Little Rock: University of Arkansas.

Capron, C., \& Duyme, M. (1989). Assessment of effects of socio-economic status on IQ in a full cross-fostering study. Nature, $340,552-554$.

Chasnoff, I. J., Anson, A., Hatcher, R., Stenson, H., laukea, K., \& Randolph, L. (1998). Prenatal exposure to cocaine and other drugs. Outcome at four to six years. Annals of the New York Academy of Sciences, 846, 314-328.

Davis EP. Bruce J. Gunnar MR. (2002). The anterior attention network: associations with temperament and neuroendocrine activity in 6-year-old children. Developmental Psychobiology. 40, 43-56.

Duncan J. Burgess P. Emslie H. (2002). Fluid intelligence after frontal lobe lesions. Neuropsychologia. 33,261-8. 
Duncan, G. J., Brooks-Gunn, J., \& Klebanov, P. K. (1994). Economic Deprivation and early-childhood development. Child Development, 65, 296-318.

Duncan, G. J., Yeung, W. J., Brooks-Gunn, J., \& Smirth, J. R. (1998). How much does childhood poverty affect the life chances of children? American Sociological Review, 63, 4-6-423.

Engle RW. Tuholski SW. Laughlin JE. Conway AR. (1999). Working memory, short-term memory, and general fluid intelligence: a latent-variable approach. Journal of Experimental Psychology: General. 128, 309-31.

Farah, M.J., Savage, J., Brodsky, N.L., Shera, D., Malamud, E., Giannetta, J. \& Hurt, H. Association of socioeconomic status with neurocognitive development. (2004) Pediatric Research, suppl.

Farah, M.J., Savage, J.,., Shera, D., Brodsky, N.L, Malamud, E., Giannetta, J. \& Hurt, H. Childhood poverty: Selective correlations with neurocognitive development. Submitted for publication.

Fukuyama, F. (2002). Our Posthuman Future: Consequences of the Biotechnology Revolution. New York: Farrar, Straus and Giroux. 
Gray JR. Chabris CF. Braver TS. (2003). Neural mechanisms of general fluid intelligence. Nature Neuroscience. 6, 316-22.

Hurt, H., Brodsky, N. L., Betancourt, L., Braitman, L., Malmud, E., \& Giannetta, J. (1995). Cocaine exposed children: Follow-up at 30 months. Developmental and Behavioral Pediatrics, 16, 29-35.

Hurt, H., Malmud, E., Braitman, L., Betancourt, L. M., Brodsky, N. L., \& Giannetta, J. M. (1998). Inner-city achievers: who are they? Archives of Pediatric and Adolescent Medicine, 152, 993-997.

Kass, L. (2003). Beyond Therapy -- Biotechnology and the Pursuit of Happiness. New York: Harper Collins.

Lewis, O. (1965). La Vida. NY: Random House.

Lupien, S. J., S. King, et al. (2001). Can poverty get under your skin? Basal cortisol levels and cognitive function in children from low and high socioeconomic status. Development and Psychopathology 13, 653-676.

Marx, K. (1976 (first published in 1867)). Capital (Vol. I). NY: Penguin. 
Mayes, L.C. (2002). A behavioral teratogenic model of the impact of prenatal cocaine exposure on arousal regulatory systems. Neurotoxicology and Teratology, 24, 385-395.

McCloyd, V. C. (1998). Socioeconomic Disadvantage and Child Development. American Psychologist, 53, 185-204.

McEwen, B. S. (2000). The neurobiology of stress: from serendipity to clinical relevance. Brain Research, 886, 172-189.

Meaney, M. J., Diorio, J., Francis, D., \& al, e. (1996). Early environmental regulation of forebrain glucocoricoid receptor gene expression: implications for adrenocortical responses to stress. Developmental Neuroscience, 18, 49-72.

Noble, K. G., Norman, M. F., \& Farah, M. J. (2005). Neurocognitive correlates of socioeconomic status in kindergarten children. Developmental Science, 8, 74-87.

Ricciuti, H. N. Nutrition and mental development. Current Directions in Psychological Science, 2, 43-46, 1993

Schiff, M., \& Lewontin, R. (1986). Education and class: The irrelevance of IQ genetic studies. Oxford: Clarendon Press.

Sigman, M. (1995). Nutrition and child development. Current Directions in Psychological 
Science 4: 52-55.

Turkheimer E., Haley A., Waldron M., D'Onofrio B. \& Gottesman II. (2003).

Socioeconomic status modifies heritability of IQ in young children. Psychological Science. $14,623-8$.

Turner RJ. Avison WR. (2003). Status variations in stress exposure: implications for the interpretation of research on race, socioeconomic status, and gender. Journal of Health \& Social Behavior. 44:488-505.

van Praag H. Kempermann G. Gage FH. (2000) Neural consequences of environmental enrichment. Nature Reviews Neuroscience. 1, 191-8, 2000

Vidaeff, A.C. \& Mastrobattista, J.M. (2003). In utero cocaine exposure: a thorny mix of science and mythology. American Journal of Perinatalogy, 20, 165-172.

Volkmar FR. Greenough WT. Rearing complexity affects branching of dendrites in the visual cortex of the rat. Science. 176):1145-7, 1972

Weber, M. (1961 first published in 1923). General Economic History: Collier. 